\title{
Application of the CO-CRS method for seismic data regularization
}

\author{
Tainá Souza (UNICAMP), Tiago Barros (UFRN), and Renato Lopes (UNICAMP)
}

Copyright 2019, SBGf - Sociedade Brasileira de Geofísica.

This paper was prepared for presentation at the $16^{\text {th }}$ International Congress of the Brazilian Geophysical Society, held in Rio de Janeiro, Brazil, August 19-22, 2019.

Contents of this paper were reviewed by the Technical Committee of the $16^{\text {th }}$ Sixteenth International Congress of the Brazilian Geophysical Society and do no necessarily represent any position of the SBGf, its officers or members. Electronic reproduction or storage of any part of this paper for commercial purposes without the written consent of The Brazilian Geophysical Society is prohibited.

\begin{abstract}
Reflection seismic is a technique widely used for geophysical studies of petroleum. However, during the seismic acquisition, operational obstacles cause the acquired seismic data to be irregular, containing distortions and gaps. This irregular sampling impairs several stages of seismic processing such as imaging, elimination of multiple, migration and inversion. Interpolation and regularization methods can solve both missing data and irregular sampling. Thereby, the regularization of seismic data is an important problem to be addressed. As we intend to show in this work, the common reflection surface method for common offset (CO-CRS) with global attributes search strategy is a good alternative for regularization, since it can reconstruct traces from the acquired data and also is capable of improving the definition of reflection events existing in the data.
\end{abstract}

\section{Introduction}

Irregular sampling of seismic data has been a problem for several stages of seismic processing, because during the seismic acquisition, operational obstacles, such as physical and budgetary constraints, make it difficult to survey in a regular mesh, add noise and generate distortions, therefore, the data are generally irregular (Moraes, 2004), (Vasconcelos 2015).

Because of the irregular sampling and gaps in the geometry of seismic data, artifacts are generated in prestacking images. And the effectiveness of imaging multiple subtraction algorithms is limited and resulting migrated images present many noises (Schonewille et al., 2009), (Trad, 2008). The low quality of seismic data also leads to the loss of the benefits and effectiveness of imaging techniques, such as pre-stacking migration and wave inversion (Abakumov et al. 2018).

For this reason, regularization of irregularly sampled and inadequate seismic data is an essential part of data preprocessing flows that must occur before the imaging and inversion phases (de Medeiros Trindade et al. 2017).

Regularization is mostly applied to solve two major issues: missing data and irregular sampling. Usually, methods based on wave equations and signal analysis are used. However, these methods present the disadvantages of changing the data and are also dependent of a velocity model.
Then appears, as an alternative to avoiding the above problems, methods based on the common reflection surface (CRS) technique, which describes the kinematics of the measured wavefield from attributes automatically obtained (Vasconcelos, 2015). And a good option for data regularization is the common reflection surface method for common offset (CO-CRS). This method can generate prestacked sections with high signal-to-noise ratio and good continuity of reflection events. Thus being practical for regularization of low quality seismic data and being able to provide a better input data for further processing steps.

The CO-CRS stacking operator approximates finite-offset reflection events in the vicinity of a given finite-offset central ray (Zhang et al. 2001). Therefore, in irregular data with null traces, the CO-CRS method can reconstruct these missing traces from the acquired data. And it can regularize any section of the pre-stacked data.

As a way of determining the CO-CRS attributes, the global search techniques stand out, because, as shown by Garabito et al. (2012), global optimization strategies for parameter stacking speed up computational costs. Moreover, in the global search, a greater number of traces are used, resulting in a more accurate approximation for the CO-CRS traveltime, which makes the coherence values less affected by noise and low fold acquisitions (Barros et al. 2015).

Still, according to Garabito et al. (2016), the simultaneous search for attributes minimizes errors and generates accurate values of CRS attributes, since the determination in several steps causes cumulative errors and the approximations that are made can generate problems in the final's construction of the pre-stacking data. Thus, the CO-CRS method with global search was chosen to be used in this work.

\section{Theory}

\section{Common Offset Common Reflection Surface}

The CO-CRS method has a stacking operator that uses the total volume of multi-coverage data during the imaging process (Bergler, 2001). Other methods, such as the Common Midpoint (Mayne 1962), use only one family of data. The CO-CRS is based on a traveltime approximation and parameterized by five attributes (Zhang et al. 2001). The goal of this technique is to synthesize a pre-stacking data, from the determination of stacking parameters for each time sample (Bergler, 2001).

The hyperbolic CO-CRS traveltime formula was developed by considering the rays in the paraxial vicinity of a central ray of the source $s$ to the receiver $r$ (Figure 1 . 


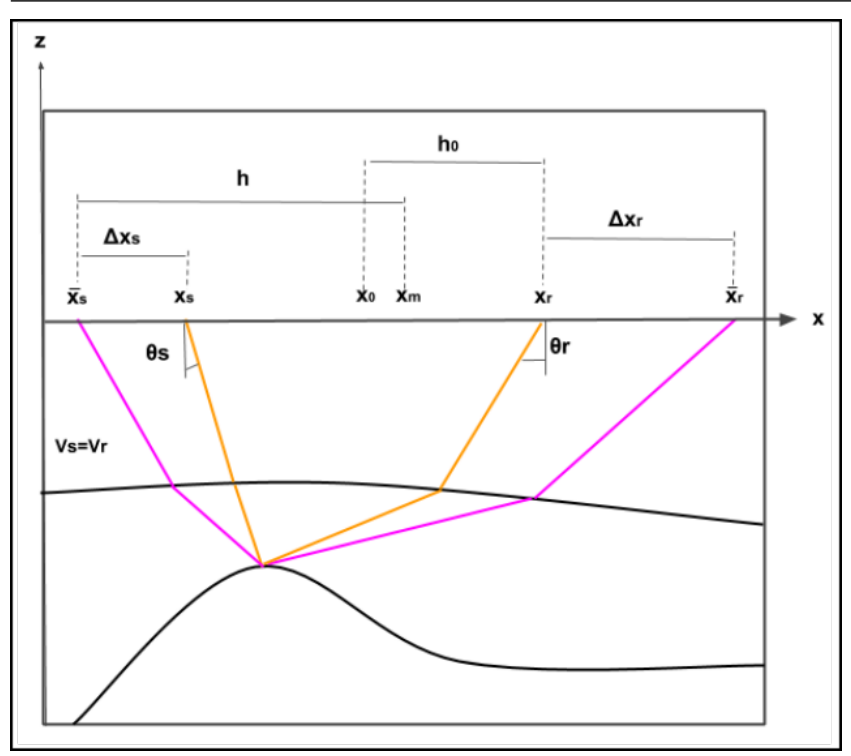

Figure 1 - Representation of a 2D model of layers with constant velocities. Source and receiver arranged in a horizontal seismic line, central ray identified with the orange color and with coordinates $x_{0}$ and $h_{0}$ and paraxial ray with pink color and coordinates $x_{m}$ and $h$ (Modified from Zhang, 2001).

The $2 \mathrm{D}$ version of the equation is shown below:

$$
\begin{aligned}
T^{2}\left(\Delta x_{m}, \Delta h\right) & =\left[t_{0}+\left(\frac{\sin \left(\theta_{r}\right)}{v_{r}}-\frac{\sin \left(\theta_{s}\right)}{v_{s}}\right) \Delta x_{m}\right. \\
& \left.+\left(\frac{\sin \left(\theta_{r}\right)}{v_{r}}-\frac{\sin \left(\theta_{s}\right)}{v_{s}}\right) \Delta h\right]^{2} \\
& +t_{0}\left[\left(4 K_{1}-3 K_{3}\right) \frac{\cos ^{2}\left(\theta_{r}\right)}{v_{r}}-K_{2} \frac{\cos ^{2}\left(\theta_{s}\right)}{v_{s}}\right] \Delta x_{m}^{2} \\
& +t_{0}\left[K_{3} \frac{\cos ^{2}\left(\theta_{r}\right)}{v_{r}}-K_{2} \frac{\cos ^{2}\left(\theta_{s}\right)}{v_{s}}\right] \Delta h^{2} \\
& +2 t_{0}\left[K_{3} \frac{\cos ^{2}\left(\theta_{r}\right)}{v_{r}}+K_{2} \frac{\cos ^{2}\left(\theta_{s}\right)}{v_{s}}\right] \Delta h \Delta x_{m}
\end{aligned}
$$

where the variable $\Delta x_{m}=x_{m}-x_{0}$ is the midpoint displacement, $\Delta h=h-h_{0}$ is the half-offset displacement, $v_{s}$ is the near-surface velocity at the source, related to the central ray and $v_{r}$ is the near-surface velocity at the receiver, related to the central ray. The five attributes determined from the pre-stack data are defined as incidence and curvature angles. $\theta_{s}$ is the incidence angle between the central ray and the $z$-axis at the source, while $\theta_{r}$ is the incidence angle at the receiver. $K_{1}, K_{2}$ e $K_{3}$ are the three wavefront curvatures associated with the central ray from source to receiver.

The global attributes search strategy consists of the simultaneous search of the CO-CRS attributes. To determine the attributes from the pre-stack data, the search is based on an optimization algorithm that searches for the global optimum, that is, the value of the parameters that leads to greater coherence Garabito et al., 2013).
In this approach, the coherence is evaluated for different combinations of the five attributes.

Considering that the correct parameters have been found, all the traces in the defined time refer to the same reflection event, with coherent or aligned samples. The CO-CRS attributes are estimated independently, at each point defined by time $t_{0}$ and by the coordinates $\left(x_{0}, h_{0}\right)$ (Barros et al. 2016).

Because it is a time-consuming and costly strategy, if solved by a brute force search on a five-dimensional grid, Barros et. al (2016) proposed the use of the global optimization algorithm differential evolution (DE), for the estimation of the CO-CRS parameters.

\section{Differential Evolution}

The DE algorithm can be defined as a direct parallel search method for continuous spatial variables. For each generation $G$, DE uses $N_{P}$ vectors of dimension $D$, $\boldsymbol{x}_{i, G}, \quad i=1, \ldots, N_{P}$, in which each coordinate of each vector contains a possible value for the $D$ parameters that must be estimated.

The initial vector population is chosen randomly, which should cover the search space relatively uniformly and guarantee the diversity among the individuals of the population. Three important operations are performed in the DE method: mutation, crossover and selection. The mutation is the first operation and is given by creating new vector parameters generated by adding the balanced difference between two population vectors and a third vector. Crossing occurs through the generation of an experimental vector created by the combination between the mutated vector parameters and another predetermined vector (target vector). Finally, in the selection, the target vector is replaced by the experimental vector if the value of the cost function for the experimental vector is greater than the value of the target vector (Barros et al. 2015).

The cost function used for the global CO-CRS search strategy is the measure of semblance and the vector parameters contains the attributes $\theta_{s}, \theta_{r}, K_{1}, K_{2}, K_{3}$, being the length $D=5$ (Barros 2018).

DE has the advantage of simple parametrization and a good and fast convergence rate. And it is desired to search for the five parameters of the CO-CRS simultaneously, but it may be too time-consuming. Thus, the application of the DE in the estimation of the attributes allows in a more practical way, the use of the global search strategy (Barros et al. 2016).

\section{Numerical experiments}

We performed tests with the CO-CRS global search in synthetic and real datasets, setting the following parameters for DE in all the tests: $D=5, N_{p}=60$ and $G=$ 400. Search apertures values are different for each dataset and are described in the section below.

\section{Synthetic data}

The selected synthetic data present minimum offset equal to $10 \mathrm{~m}$, maximum offset of $1790 \mathrm{~m}, 269$ CDPs and time sample interval of $\mathrm{dt}=0.004 \mathrm{~s}$. We added random noise to the data to reduce the signal-to-noise ratio $(S N R=20)$. We selected the common offset gather with an offset equal 
to $270 \mathrm{~m}$. The aperture values applied were: $50 \mathrm{~m}$ for midpoint and $100 \mathrm{~m}$ for offset (Figure 2 e 4).

Analyzing the obtained results, it can be observed that even with the presence of noise, the data presented a good enhancement in the definition of the traces, mainly below the horizontal reflector (Figura $3 \mathrm{e} 5$.

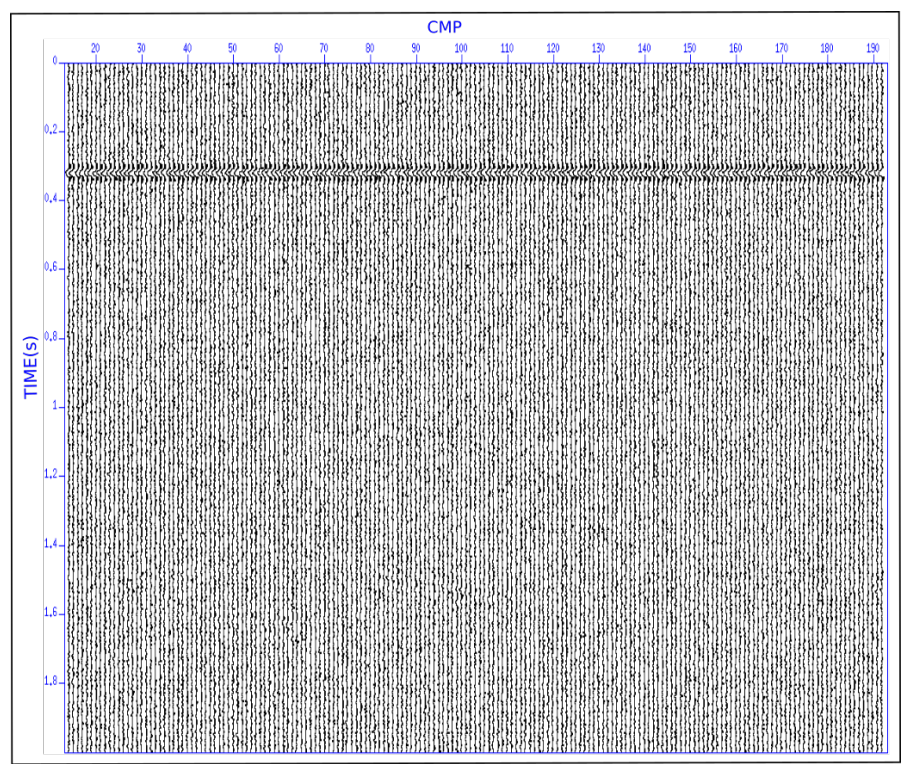

Figure 2 - Noisy traces from the commom offset gather $270 \mathrm{~m}$ of the synthetic data with noise.

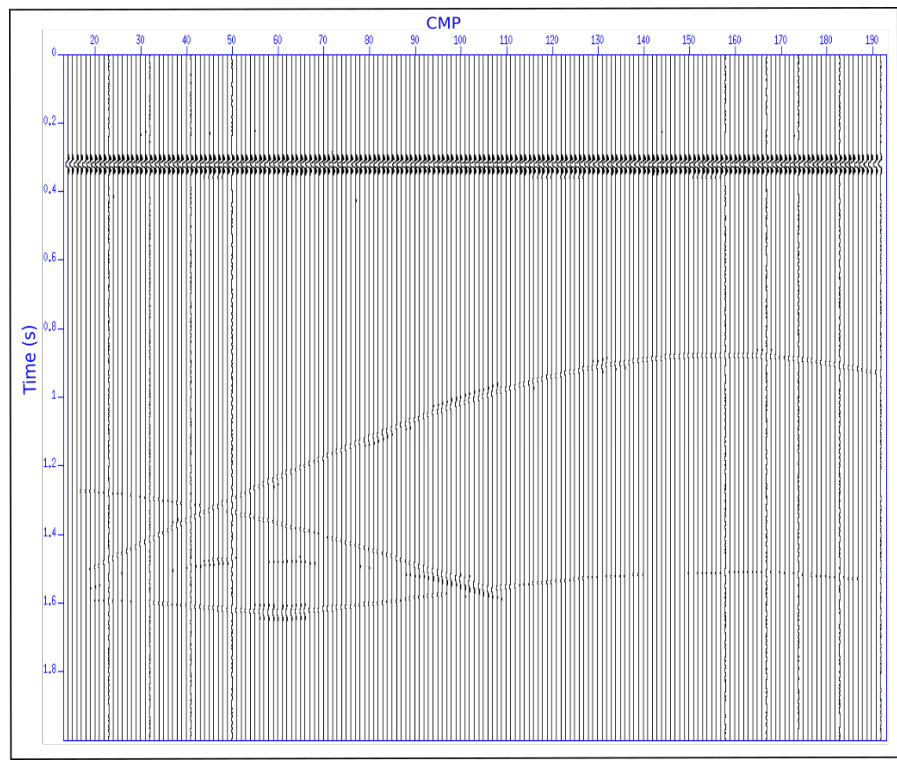

Figure 3 - Regularized traces from the commom offset gather $270 \mathrm{~m}$ of the synthetic data.

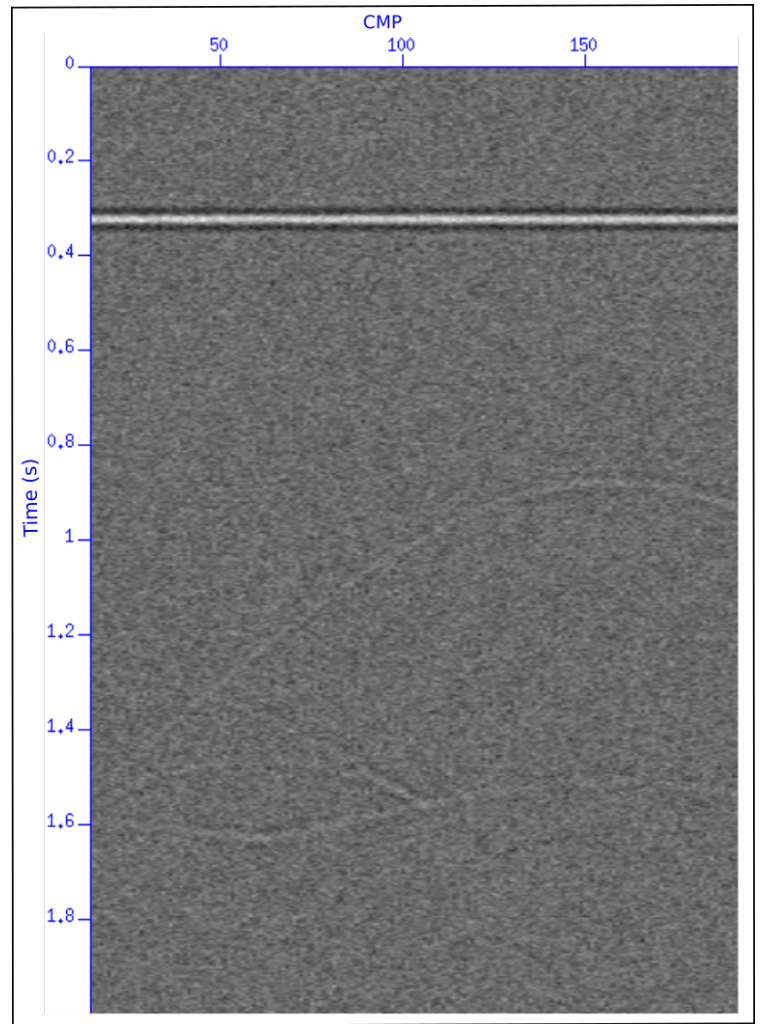

Figure 4 - Commom offset gather $270 \mathrm{~m}$ of the synthetic data with noise.

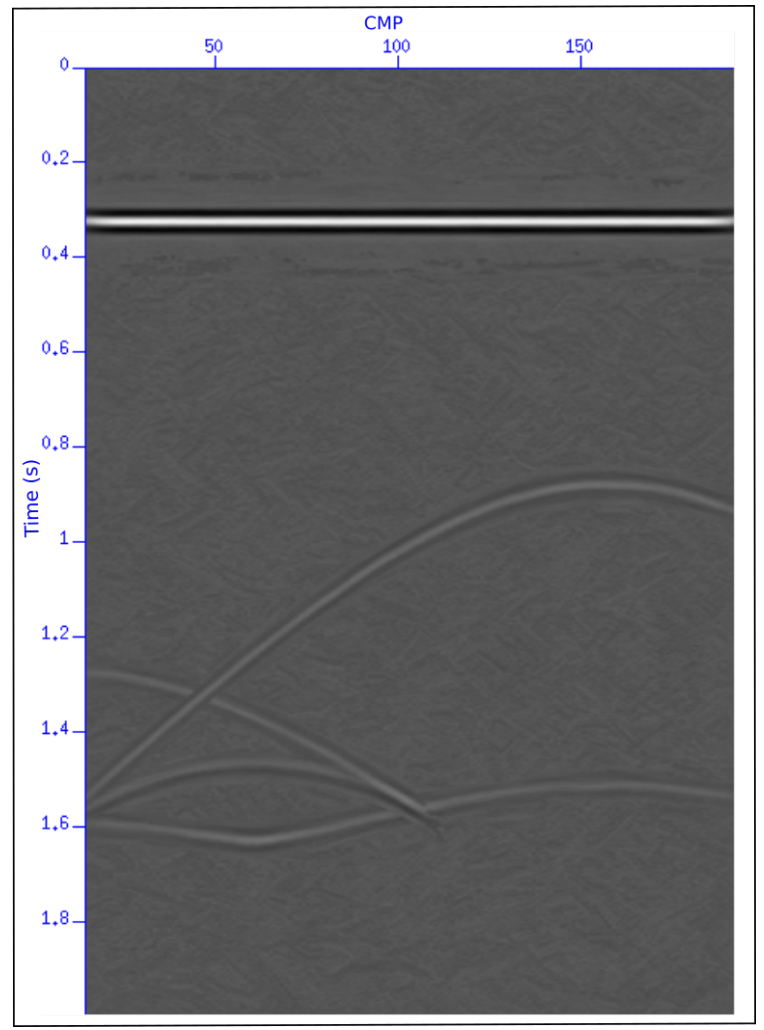

Figure 5 - Commom offset gather $270 \mathrm{~m}$ of the synthetic data regularized. 
Posteriorly, we randomly eliminated some traces of the synthetic data and selected a new common offset gather with a coordinate equal to $1790 \mathrm{~m}$. The same aperture values for offset $(100 \mathrm{~m})$ and midpoint $(50 \mathrm{~m})$ applied previously were used (Figure 6e 8. When comparing the original data and the data with deleted traces, it is possible to notice that the traces were well reconstituted and, in addition, the data were improved with the increase of the definition of reflections and diffractions (Figura $7 \mathrm{e} 9$ ).

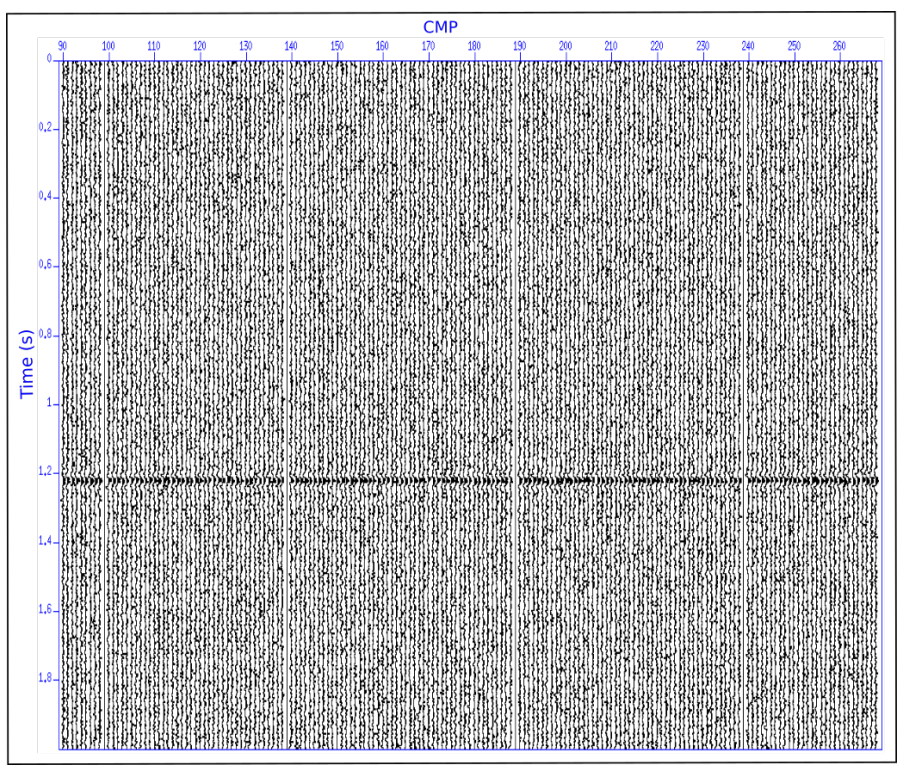

Figure 6 - Noisy traces from the commom offset gather $1790 \mathrm{~m}$ of the synthetic data with noise and eliminated traces.

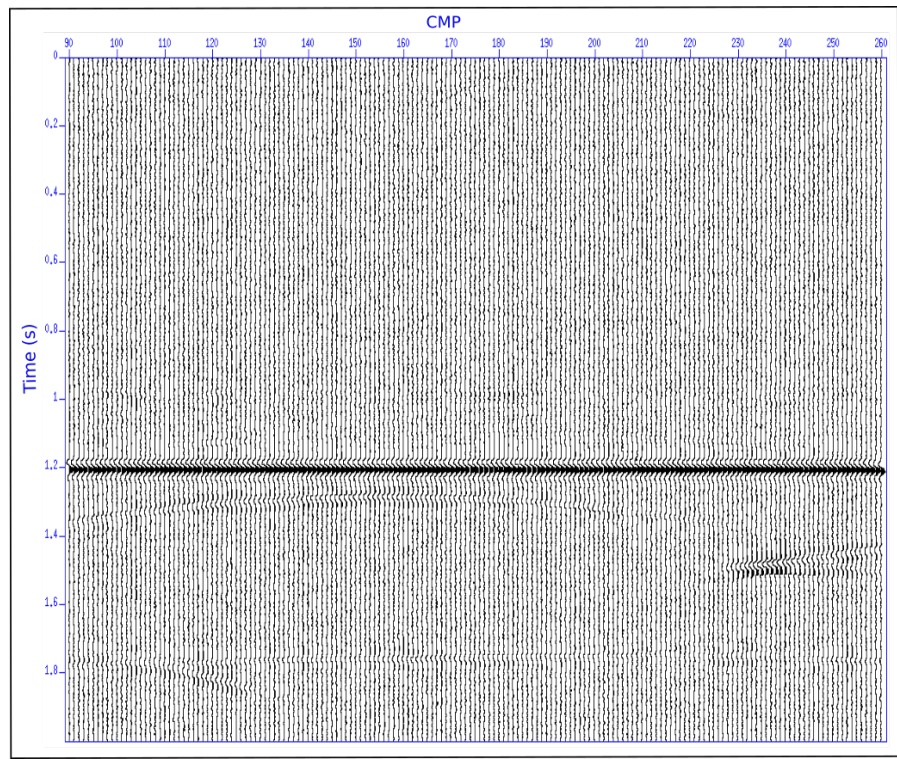

Figure 7 - Regularized traces from the commom offset gather $1790 \mathrm{~m}$ of the synthetic data.

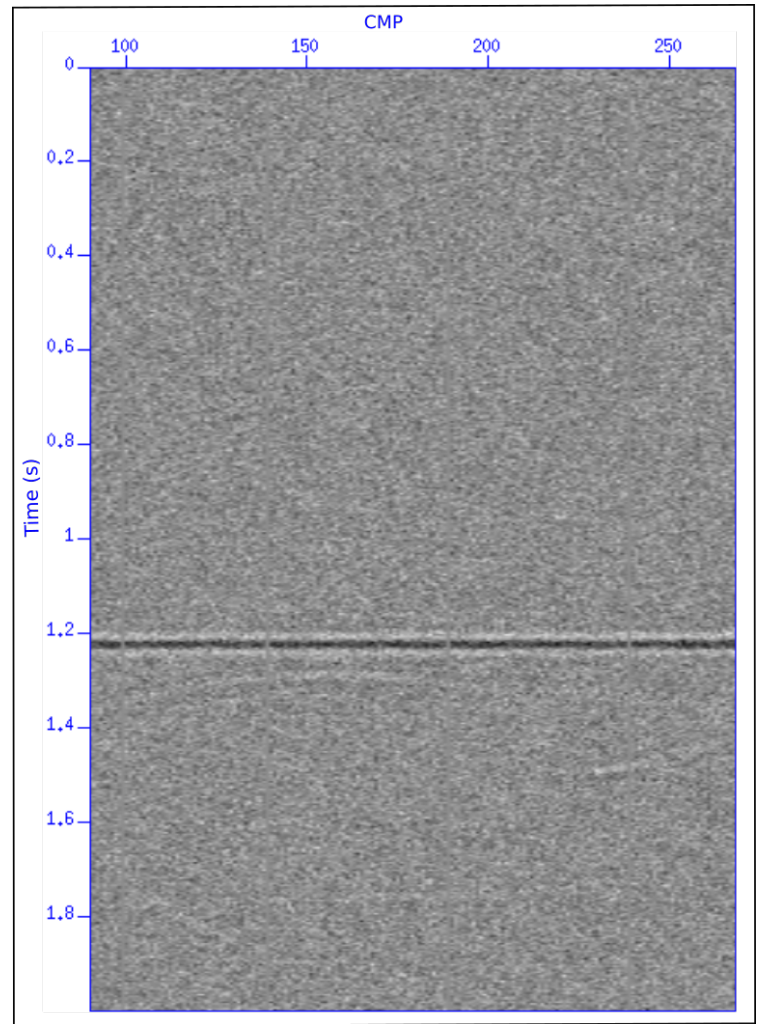

Figure 8 -Commom offset gather $1790 \mathrm{~m}$ of the synthetic data with noise.

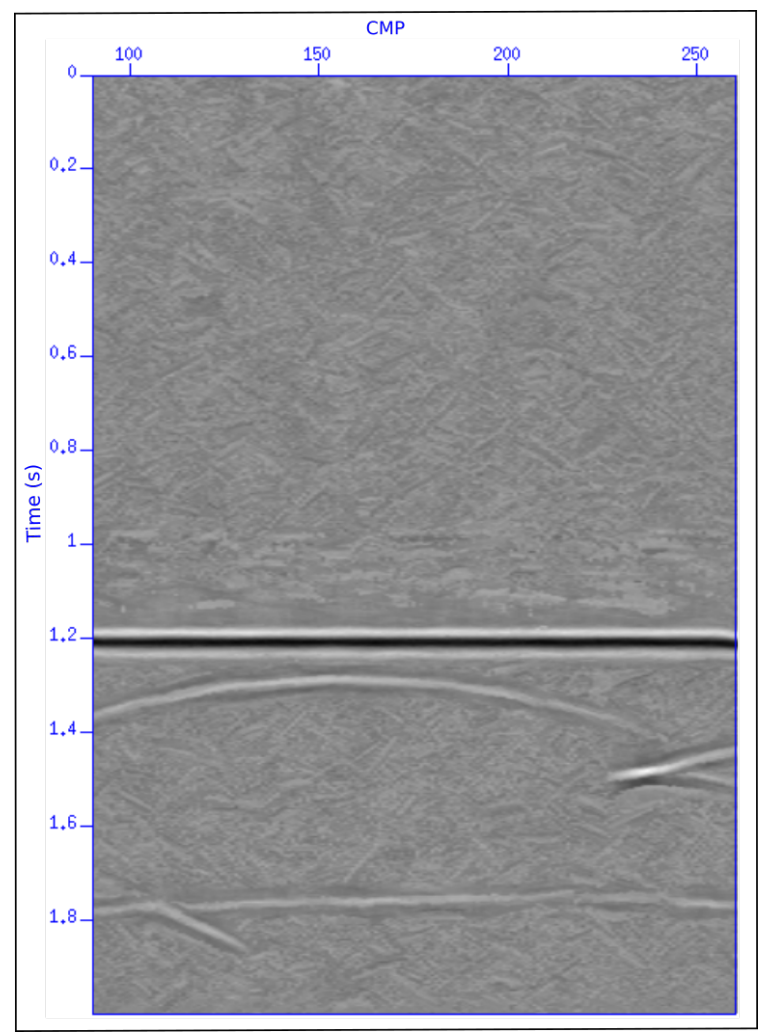

Figure 9 -Commom offset gather $1790 \mathrm{~m}$ of the synthetic data regularized. 


\section{Field data}

For the real data tests we selected a land seismic line from Alaska (USGS 2017), containing shotpoint interval equal to $135 \mathrm{~m}$, receiver interval equal to $34 \mathrm{~m}$, minimum offset equal to $-5225 \mathrm{~m}$ and the maximum offset equal to $5225 \mathrm{~m}$. The selected common offset gather was the offset -5225 $\mathrm{m}$, which contains null trace (Figure 10 and the aperture values applied were $400 \mathrm{~m}$ for midpoint and $500 \mathrm{~m}$ for offset.

The selected offset, which contained a missing trace, evidenced the CO-CRS ability of trace reconstruction, and also the improvement in the image quality. We noted an increase in the continuity and greater definition of some reflections in several regions of the regularized data and the coherent reconstruction of the null trace, indicated by the red arrows (Figura 11 .

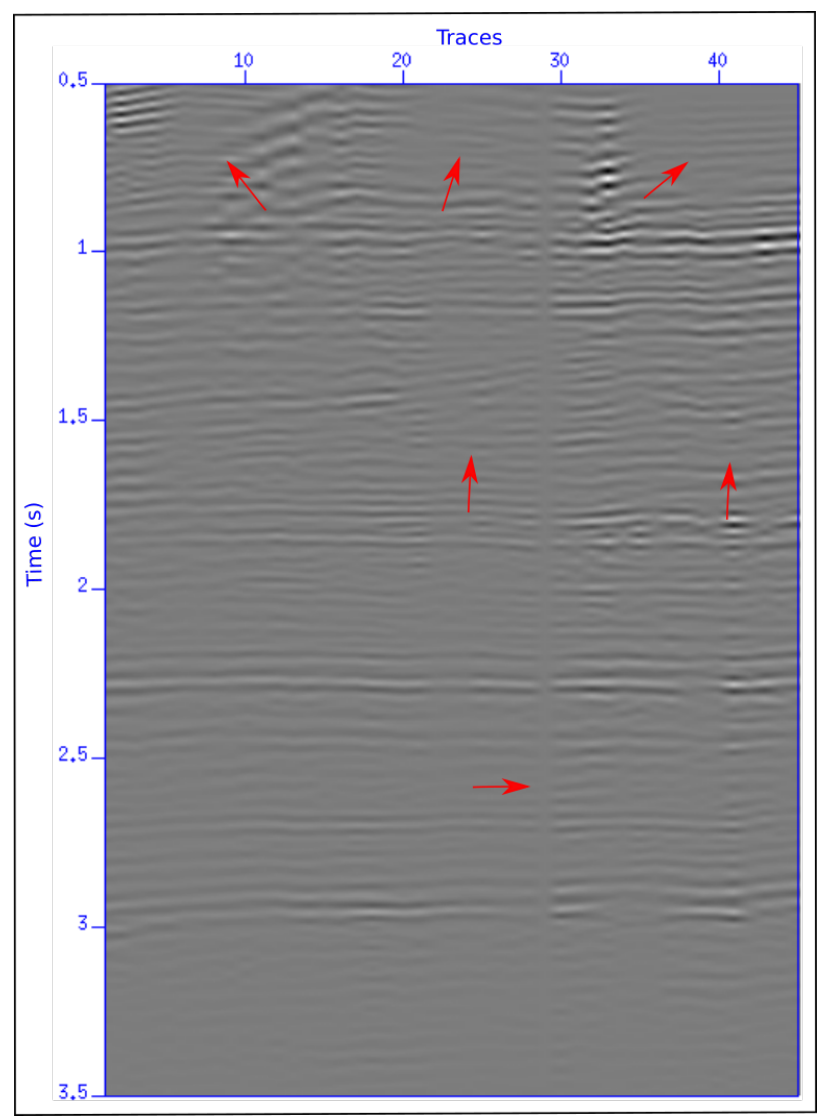

Figure 10 - Commom offset gather $-5225 \mathrm{~m}$ of the raw Alaska data.

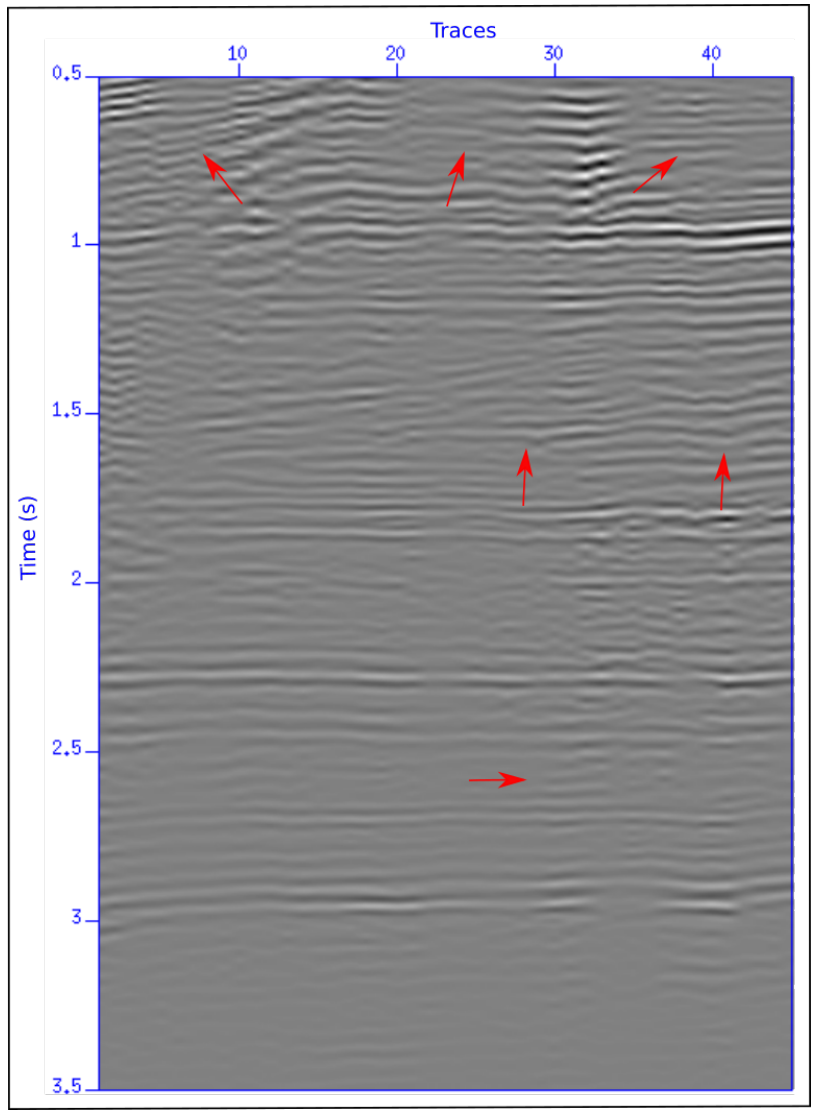

Figure 11 - Commom offset gather $-5225 \mathrm{~m}$ of the Alaska data regularized.

\section{Conclusions}

The application of the CO-CRS method demonstrated to be effective in improving the definition of the original traces, increasing the signal-to-noise ratio and evidencing the reflection, and difraction events presented in the synthetic and real datasets. The CO-CRS can also to consistently reconstruct the missing traces, in both the examples. Therefore, this method is particularly attractive for land data with low fold, and random and low frequency noise, because it can efficiently regularize irregular seismic data.

\section{Acknowledgments}

The authors thank the Brazilian research agency Coordination for the Improvement of Higher Education Personnel (CAPES) and the School of Electrical Engineering and Computing from the University of Campinas (FEEC - UNICAMP) for the support for this work.

\section{References}

Abakumov, I., B. Kashtan, and D. Gajewski, 2018, Time-lapse data enhancement and regularization with common-offset crs stack: Presented at the 80th EAGE Conference and Exhibition 2018.

Barros, T., R. Krummenauer, R. Lopes, and H. Chauris, 2016, Pre-stack data recovery through common offset crs stack with differential evolution: Presented at the 78th EAGE Conference and Exhibition 2016.

Barros, T., R. Lopes, and M. Tygel, 2015, Implementation 
aspects of eigendecomposition-based high-resolution velocity spectra: Geophysical Prospecting, 63, 99-115.

Barros, T. T. L., 2018, Contributions to the initialization of seismic slope tomography: $\mathrm{PhD}$ thesis, Faculdade de Engenharia Elétrica e Computação, Universidade de Campinas.

Bergler, S., 2001, The common-reflection-surface stack for common offset-theory and application: Master's thesis, Universität Karlsruhe (Germany).

de Medeiros Trindade, J., G. Garabito, M. D. Sacchi, and L. dos Santos Lucena, 2017, A comparison of 2d seismic data regularization with mwni and $\mathrm{mp}$ : 15th International Congress of the Brazilian Geophysical Society \& EXPOGEF, Rio de Janeiro, Brazil, 31 July3 August 2017, Brazilian Geophysical Society, 13931398.

Garabito, G., P. Stoffa, and W. Söllner, 2013, Global optimization of the common-offset crs-attributes: Synthetic and field data application: 13th International Congress of the Brazilian Geophysical Society \& EXPOGEF, Rio de Janeiro, Brazil, 26-29 August 2013, Society of Exploration Geophysicists and Brazilian Geophysical Society, 1565-1568.

Mayne, W. H., 1962, Common reflection point horizontal data stacking techniques: Geophysics, 27, 927-938.

Moraes, D., 2004, Interpolação e regularização de dados sísmicos usando a transformada de radon linear $(\tau-p) 2 d$ e 3d: PhD thesis, Faculdade de Engenharia Mecênica, Universidade de Campinas.

Schonewille, M., A. Klaedtke, A. Vigner, J. Brittan, and T. Martin, 2009, Seismic data regularization with the antialias anti-leakage fourier transform: First Break, 27.

Trad, D., 2008, Five dimensional seismic data interpolation, in SEG Technical Program Expanded Abstracts 2008: Society of Exploration Geophysicists, 978-982.

USGS, 2017, National petroleum reserve alaska.

Vasconcelos, T. W. V., 2015, Seismic data regularization based on crs attributes: Master's thesis, Faculdade de Engenharia Mecânica, Universidade de Campinas.

Zhang, Y., S. Bergler, and P. Hubral, 2001, Commonreflection-surface (crs) stack for common offset: Geophysical Prospecting, 49, 709-718. 